\title{
Aeroelastic System Development Using Proper Orthogonal Decomposition and Volterra Theory
}

\author{
David J. Lucia* and Philip S. Beran ${ }^{\dagger}$ \\ Air Force Research Laboratory \\ Walter A. Silva $\ddagger$ \\ NASA Langley Research Center
}

\begin{abstract}
This research combines Volterra theory and proper orthogonal decomposition (POD) into a hybrid methodology for reduced-order modeling of aeroelastic systems. The outcome of the method is a set of linear ordinary differential equations (ODEs) describing the modal amplitudes associated with both the structural modes and the POD basis functions for the fluid. For this research, the structural modes are sine waves of varying frequency, and the Volterra-POD approach is applied to the fluid dynamics equations. The structural modes are treated as forcing terms which are impulsed as part of the fluid model realization. Using this approach, structural and fluid operators are coupled into a single aeroelastic operator. This coupling converts a free boundary fluid problem into an initial value problem, while preserving the parameter (or parameters) of interest for sensitivity analysis. The approach is applied to an elastic panel in supersonic cross flow. The hybrid Volterra-POD approach provides a low-order fluid model in state-space form. The linear fluid model is tightly coupled with a nonlinear panel model using an implicit integration scheme. The resulting aeroelastic model provides correct limit-cycle oscillation prediction over a wide range of panel dynamic pressure values. Time integration of the reduced-order aeroelastic model is four orders of magnitude faster than the high-order solution procedure developed for this research using traditional fluid and structural solvers.
\end{abstract}

\section{Introduction}

Volterra methods ${ }^{1}$ and proper orthogonal decomposition $^{2,3}$ (POD) are two of the more prevalent reducedorder modeling (ROM) techniques well-suited to nonlinear dynamics. ${ }^{4-8}$ The application of ROM techniques to aeroelastic systems is an active area of research, motivated by the desire for faster algorithms that are well-suited to the design environment for aircraft. For example, transonic, fluid-structure interaction is a particular application of interest to both external and internal aerodynamicists because moving shock waves in the flow necessitate high-fidelity numerical flow solvers which are too cumbersome for iterative design analysis. Regardless of the application, when nonlinearities are present in either the flow field or the

*Senior Research Aerospace Engineer, Major, USAF, AFRL/VAS, Bldg 45, 2130 Eighth Street, Suite 1, WPAFB, OH 45433-7542, (david.lucia@wpafb.af.mil), AIAA Member

${ }^{\dagger}$ Principal Research Aerospace Engineer, AFRL/VASD, Bldg 146, 2210 Eighth Street, WPAFB, OH 45433-7531, (philip.beran@wpafb.af.mil), Associate Fellow of AIAA

¥Senior Research Scientist, Aeroelasticity Branch, Mail Stop 340, NASA Langley Research Center, Hampton, VA 23681-0001, Senior Member of AIAA

Copyright (C) 2003 by the American Institute of Aeronautics and Astronautics, Inc. No copyright is asserted in the United States under Title 17, U.S. Code. The U.S. Government has a royaltyfree license to exercise all rights under the copyright claimed herein for Governmental Purposes. All other rights are reserved by the copyright owner. structure, established order-reduction methods that rely on linearized dynamics are of little use.

Over the past three years, applications of POD to the Euler equations have produced reduced order aeroelastic models that properly capture aerodynamic nonlinearities. A low-order POD representation of the discrete, 2-D Euler equations ${ }^{9}$ was coupled with the von Kármán equation to simulate the dynamics of flow over a flexible panel. ${ }^{10}$ Subsequently, a new approach was taken, involving domain decomposition, that allowed LCO to be accurately simulated in the transonic regime. ${ }^{11}$ In that study, full-order and reduced order models of a small flow region containing a moving shock were decomposed from the larger flow domain. Both approaches enabled a physically consistent treatment of the aerodynamic nonlinearity. In a more recent paper, ${ }^{12}$ the original $\mathrm{POD} / \mathrm{ROM}$ methodology used for flow over an elastic panel ${ }^{10}$ was revisited to improve the temporal coupling between the aerodynamic and structural dynamic equations. Furthermore, a modal representation of the structure was employed, which permitted a more efficient formulation of the reduced-order aeroelastic system.

All of the studies mentioned above relied on a ROM technique called subspace projection for time integration of the reduced-order model. While sufficient to demonstrate the accuracy of the POD basis functions, 
subspace projection was not an efficient way to time integrate the low-order, aeroelastic ROM. Generally, four orders of magnitude reduction in fluid system degrees of freedom (DOFs) were demonstrated in the above studies. Time integrating these POD/ROMs with subspace projection generally produced about one order of magnitude improvement in compute time to accompany a much larger drop in problem order.

The applicability of POD basis functions to nonlinear problems has been documented in the literature, but a tractable nonlinear, low-order model realization procedure is a key missing link. Two techniques, Galerkin projection and direct projection, have been recently reported as having potential for obtaining nonlinear terms for POD/ROMs. ${ }^{13}$ However, the linear portion of these realization procedures is generally unstable, requiring dissipation techniques that affect model performance. The Volterra-POD approach provides a stable reduced-order equation set, and is an important advance toward achieving stable, nonlinear reduced-order models.

The hybrid Volterra-POD method was recently developed to replace subspace projection for time integration of $\mathrm{POD} / \mathrm{ROM}$ applied to compressible flow fields. ${ }^{14}$ The goal of the Volterra-POD approach was to achieve computational savings on the order of DOF reductions. This goal was achieved in the initial application, where four orders of magnitude reduction was obtained in both DOFs and compute time. To date, the hybrid Volterra-POD method has only been applied to subsonic flow-fields characterized by linear behavior, with fixed boundaries. The product of the technique was a linear, state-space system of ODEs governing the dynamics of modal coefficients corresponding to a small number of POD basis functions. The state-space realization was obtained from a set of impulse responses that were processed using the Eigensystem Realization Algorithm (ERA). ${ }^{15,16}$

This research will extend the Volterra-POD approach to supersonic flow-fields with dynamic boundary behavior. The POD-Volterra method will be applied to a two-dimensional elastic panel in inviscid, supersonic cross-flow. The Volterra-POD approach will be used to identify a low-dimensional, linear POD/ROM for the fluid. The POD/ROM will be tightly coupled to a low-dimensional, nonlinear model of the von Kármán plate equation. ${ }^{17}$ The aeroelastic response will be obtained using an implicit timeintegration scheme.

The Volterra-POD technique involves procedures that require the selection of parameters such as impulse size, several data windowing lengths, and impulse sampling frequency. The choice of POD basis affects performance as well. Some considerations for generating the POD basis include choice of base flow (the POD/ROM determines the perturbation to this base flow), snapshot collection method and sampling frequency (the method of snapshots ${ }^{18}$ will be discussed in the next section).

The research will consider two base flow cases, and two snapshot collection methods. Both uniform flow at free stream conditions, and steady-state flow over a static panel deflection will be considered as base flow cases. An aeroelastic POD basis will be generated by sampling a small portion of the time history for a baseline LCO case, which was the approach in recent applications using subspace projection for this problem. ${ }^{12}$ In addition, we will investigate using the impulse response of the fluid system to generate a POD basis. The full-system impulse response is collected as part of the Volterra-POD approach, and the impulse responses can be sampled as snapshots in lieu of the LCO time-history. Finally, we will apply POD to the structural dynamics, couple the structural POD/ROM with the fluid POD/ROM and examine performance. We will record the various parameter settings used to generate the aeroelastic POD/ROMs for each case.

The linearity of the supersonic flow-field will be examined as part of the ROM analysis. The principle of superposition applies in a linear flow-field, which enables a host of linear order-reduction techniques, including the Volterra-POD technique detailed in this paper. While the supersonic, aeroelastic flow-field is well represented by a linear fluid model, we will demonstrate that the supersonic flow-field itself is not linear in general.

The performance of the Volterra-POD aeroelastic ROMs will be quantified in accuracy, order reduction, and computational savings. A high-order, full-system representation of the problem is required for snapshot collection. The flow field and panel response for the full-system model will serve as the baseline for performance comparison. Accuracy will be quantified by comparing LCO panel response, flow-field pressure distribution on the elastic panel, LCO frequency, and LCO phase for a variety of panel dynamic pressure values. Finally, the robustness of the Volterra-POD method for predicting LCO response across a broad parameter space will also be addressed.

\section{Formulation}

This section describes the full-system aeroelastic model, introduces POD, and overviews Volterra methods. In addition, we fully develop the Volterra-POD approach and the synthesis of aeroelastic ROMs.

\section{Structural Dynamics Equations}

Two-dimensional flow over a semi-infinite, pinned panel of length $L$ is considered. Panel dynamics are computed with von Kármán's large-deflection plate equation, which is placed in nondimensional form using aerodynamic scales $L$ and $u_{\infty}{ }^{19}(0<x<1)$ :

$$
\frac{\mu}{\lambda} \frac{\partial^{4} w}{\partial x^{4}}-N_{x} \frac{\partial^{2} w}{\partial x^{2}}+\frac{\partial^{2} w}{\partial t^{2}}=\mu\left(\frac{1}{\gamma M_{\infty}^{2}}-p\right)
$$




$$
N_{x} \equiv \frac{6 \mu}{\lambda}\left(\frac{h}{L}\right)^{-2}\left(1-\nu^{2}\right) \int_{0}^{1}\left(\frac{\partial w}{\partial x}\right)^{2} d x .
$$

The nonlinear, in-plane load in Eqn. (2), serves to limit panel deflections $w(x, t)$ induced by fluid-structure interaction. Here, the load is assumed to be distributed uniformally over the panel. ${ }^{17}$ Equation (1) is comparable to similar formulations found in the literature, ${ }^{17}$ although $w_{d}$ and $t_{d}$ are scaled by $h$ and $\left(\rho_{s} h L^{4}\right)^{1 / 2}$, respectively. Two pinned boundary conditions are enforced at the panel's endpoints: $w=0$ and $\frac{\partial^{2} w}{\partial x^{2}}=0$.

A modal solution for the deflection $w(x, t)$ is assumed:

$$
w(x, t)=\sum_{i=1}^{m_{s}} a_{i}(t) \sin (i \pi x),
$$

where $m_{s}$ is the number of structural modes retained, and the modal amplitudes $a_{i}$ vary in time and are collocated in the array $\boldsymbol{a}$. The Galerkin method is used to obtain a low-order set of ordinary-differential equations describing the behavior of $a_{i} .{ }^{17}$ First, Eqn. (3) is substituted into Eqn. (1). The resulting expression is then integrated, following pre-multiplication by $\sin (i \pi x)$, to yield $\left(i=1, \ldots, m_{s}\right)$

$\frac{1}{2} \ddot{a}_{i}+\frac{\mu(i \pi)^{4}}{2 \lambda} a_{i}+\frac{6 \mu}{\lambda}\left(\frac{h}{L}\right)^{-2}\left(1-\nu^{2}\right) \alpha \frac{(i \pi)^{2}}{2} a_{i}=\mu P_{i}$,

where $\alpha \equiv \sum_{r} a_{r}^{2} \frac{(r \pi)^{2}}{2}$ and

$$
P_{i} \equiv \int_{0}^{1}\left(\frac{1}{\gamma M_{\infty}^{2}}-p\right) \sin (i \pi x) d x .
$$

The projected pressure components, $P_{i}$, are integrated from the aerodynamic solution with the midpoint rule, using flowfield pressures obtained at grid points on the panel surface. ${ }^{12}$ The aerodynamic equations, their discretization, and their solution are discussed in later sections. While equivalent to other formulations in the literature, Eq. (4) has two notable distinctions. First, the different form of scaling described above alters equation coefficients, and, second, an expression relating $p$ to the state of the panel is not assumed. ${ }^{12}$

The structural dynamics equation Eqn. (4) is placed in first-order form by introducing a mode speed array, $\boldsymbol{b}$, such that $\dot{a}_{i}=b_{i}$,

$$
\dot{b}_{i}=-\left[\frac{\mu(i \pi)^{4}}{\lambda}+\frac{6 \mu}{\lambda}\left(\frac{L i \pi}{h}\right)^{2}\left(1-\nu^{2}\right) \alpha\right] a_{i}+2 \mu P_{i} .
$$

The mode speeds and amplitudes are collocated into a structural solution array, $\boldsymbol{Y}_{s}$, leading to a general form of the structural equation:

$$
\begin{aligned}
\boldsymbol{Y}_{s} & =[\boldsymbol{b}, \boldsymbol{a}]^{T} \\
\dot{\boldsymbol{Y}}_{\boldsymbol{s}} & =R_{s}\left(\boldsymbol{Y}_{\boldsymbol{s}}, \boldsymbol{P} ; \mu, \lambda, h / L\right) .
\end{aligned}
$$

\section{Fluid Dynamics}

The dynamics of inviscid fluid flows are governed by the Euler equations. The two-dimensional Euler equations are given below in strong conservation form: ${ }^{20}$

$$
\begin{aligned}
\frac{\partial U}{\partial t} & +\frac{\partial E(U)}{\partial x}+\frac{\partial F(U)}{\partial y}=0, \\
U(\boldsymbol{x}, t) & =\left[\begin{array}{c}
\rho \\
m_{x} \\
m_{y} \\
E_{T}
\end{array}\right],
\end{aligned}
$$

where $\rho, m_{x}, m_{y}$ and $E_{T}$ are functions of space and time. Since we assume an ideal gas for our applications, this equation set can be closed using the ideal gas law.

The solution of the Euler equations can be approximated using either finite-difference, finite-volume, or finite-element techniques. To do this, the spatial domain is discretized, and the flow variables in $U(\boldsymbol{x}, t)$ at each discrete location are collocated into a column vector $\boldsymbol{U}(t)$. Time integration across the computational mesh is used to obtain flow solutions.

Since the Euler equations are linear in the time derivative, and quasi-linear in the spatial derivative ${ }^{20,21}$ the spatial derivatives and the time derivatives in Eqn. (8a) can be separated to form an evolutionary system. To accomplish this, the spatial derivatives of the flux terms $\frac{\partial E}{\partial x}$ and $\frac{\partial F}{\partial y}$ are grouped to form a nonlinear operator $R$ acting on the set of fluid variables. The fluid dynamics from Eqn. (8a) can then be expressed as

$$
\frac{d U(\boldsymbol{x}, t)}{d t}=R(U(\boldsymbol{x}, t)) .
$$

When discretized this expression takes the form

$$
\frac{d \boldsymbol{U}(t)}{d t}=R(\boldsymbol{U}(t)) .
$$

Equation (10) is referred to as the full-system dynamics.

A finite-volume scheme was the basis for the fullorder solver used in this research, which approximated the integral form of the Euler equations:

$$
\frac{d}{d t} \int_{\mathrm{V}} U d \mathcal{V}+\int_{\partial \mathrm{V}}(E \widehat{\imath}+F \widehat{\jmath}) \cdot d \overline{\mathcal{S}}=0
$$

The grid points in the computational mesh described earlier were used to form corners for cells. For each cell, the integral form of the Euler equations reduced to the following, assuming no grid deformation:

$$
\frac{d}{d t} U_{i, j}+\sum_{\text {sides }}\left(E_{i, j} \widehat{\imath}+F_{i, j} \widehat{\jmath}\right) \cdot \frac{d \overline{\mathcal{S}}_{i, j}}{d \mathcal{A}_{i, j}}=0 .
$$

The flux terms $E_{i, j}$ and $F_{i, j}$ were computed using second-order Roe averaging, ${ }^{20}$ and the flow variables 
$U_{i, j}$ were evaluated as cell averages. Time integration across the computational mesh was used to obtain flow solutions. This was accomplished with a first-orderaccurate, forward Euler approximation.

External boundaries were handled with ghost cells. The fluid values for the ghost cells at the far field boundaries were determined using characteristic boundary conditions. ${ }^{20}$ The bump-surface was modeled using a transpiration approximation..$^{22}$ The finitevolume fluid solver and the transpiration boundary condition were validated using a combination of theory and experimental data. Subsonic performance was validated using wind-tunnel data. ${ }^{23}$ Supersonic performance was validated using oblique shock theory. ${ }^{24}$ Time-accurate performance was validated by correctly predicting the vortex shedding frequency from a cylinder in cross flow.

\section{Time Integration of the Coupled Full-Order Equations}

The systems of discretized fluid dynamic equations, $\boldsymbol{U}(t)$, and modal structural equations, $\boldsymbol{Y}_{s}$, are combined into a single time-dependent system representative of the complete interaction between structure and inviscid flow. Time integration proceeds in two steps, assuming an $\mathcal{O}(\Delta t)$ lag in the synchronization of fluid and structure. First, the structural variables are updated from time level $n$ to $n+1$ using a CrankNicolson procedure to be described below (but limited here to only structural variables). During this step, the pressures known at grid points on the panel surface are considered frozen. In the second step, the aerodynamic variables are explicitly updated using only structural variables defined at time level $n$.

\section{Grid Generation and Time Step}

The flow is simulated over a physical domain of length $D_{L}$, centered about $x=0$, and height $D_{H}$. The domain is discretized using $I$ nodes in the streamwise direction and $J$ nodes normal to the panel. Indices $i$ $(1 \leq i \leq I)$ and $j(1 \leq j \leq J)$ are used to denote grid points comprising corners of cells for the finite-volume scheme. Grid points are clustered in the direction normal to the panel at the panel surface, with minimum spacing denoted by $\Delta_{\text {wall }}$. The spacing of grid points is specified to grow geometrically with $j$ from the panel boundary. In the streamwise direction, the node spacing is chosen to be uniform over the deforming panel segment (coincident with the structural grid), while growing geometrically upstream of the leading edge (positioned at $\left.i=I_{L E}\right)$ and downstream of the trailing edge (positioned at $i=I_{T E}$ ). Calculations are carried out with a baseline grid given by the following: $I=141, J=116, D_{L}=50, D_{H}=25, I_{L E}=45$, $I_{T E}=97$, and $\Delta_{\text {wall }}=0.0125$.

\section{Proper Orthogonal Decomposition}

POD is a technique to identify a small number of basis functions that adequately describe the behavior of the full-system dynamics (Eqn. (10)) across some parameter space of interest. A summary of POD as it applies to a spatially-discretized flow field follows. A detailed description of POD is available in the literature. ${ }^{4,8}$ For simplicity, consider only one fluid variable, $\mathrm{w}(\boldsymbol{x}, t)$, which when spatially descritized using $N$ nodes is denoted $\mathbf{w}(t)$. For this fluid variable, the full-system dynamics in Eqn. (10) is expressed as

$$
\frac{d \mathbf{w}}{d t}=R_{w}(\mathbf{w}) .
$$

Spectral methods approximate the solution $\mathrm{w}(\boldsymbol{X}, t)$ as

$$
\mathrm{w}(\boldsymbol{x}, t) \approx \sum_{k=1}^{M} a_{k}(t) \phi_{k}(\boldsymbol{x}) .
$$

When the domain is spatially discretized, $\phi_{k}(\boldsymbol{x})$ becomes a vector $\phi_{k}$, and the following relation applies:

$$
\mathbf{w}(t) \approx \sum_{k=1}^{M} a_{k}(t) \phi_{k} .
$$

The set of vectors $\left\{\phi_{k}\right\}$ are discrete basis functions corresponding to the computational mesh defined for the numerical solver. The set $\left\{a_{k}\right\}$ are the modal coefficients, and Eqn. (15) can be represented using matrix algebra. The fluid modes comprise columns of a modal matrix $\Phi$, and the coefficients are collocated into a column vector $\widehat{\mathbf{w}}(t)$. POD produces a linear transformation $\Phi$ between the full-order solution, $\mathbf{w}$, and the reduced-order solution, $\widehat{\mathbf{w}}$ :

$$
\mathbf{w}(t)=\mathbf{W}_{0}+\Phi \widehat{\mathbf{w}}(t) .
$$

The reduced-order variable $\widehat{\mathbf{w}}(t)$ represents deviations of $\mathbf{w}(t)$ from a base solution $\mathbf{W}_{0}$. The subtraction of $\mathbf{W}_{0}$ will result in zero-valued boundaries for the POD modes wherever constant boundary conditions occur on the domain.

$\Phi$ is constructed by collecting observations of the solution $\mathbf{w}(t)-\mathbf{W}_{0}$ at different time intervals throughout the time integration of the full-system dynamics. These observations are called snapshots ${ }^{18}$ and are generally collected to provide a good variety of flow field dynamics while minimizing linear dependence. The snapshot generation procedure is sometimes referred to as POD training. ${ }^{8}$

A total of $Q$ snapshots are collected from the fullsystem dynamics. These are vectors of length $N$. The set of snapshots describe a linear space that is used to approximate both the domain and the range of the nonlinear operator $R_{w}$. The linear space is defined by the span of the snapshots. ${ }^{25}$ POD identifies a new basis for this linear space that is optimally convergent ${ }^{4}$ 
in the sense that no other set of basis functions will capture as much energy in as few dimensions as the POD basis functions. To identify the POD basis, the snapshots are compiled into an $N \times Q$ matrix $S$, known as the snapshot matrix. The mapping function $\Phi$ is then developed using

$$
\begin{aligned}
S^{T} S V & =V \Lambda, \\
\Phi & =S V .
\end{aligned}
$$

Here $V$ is the matrix of eigenvectors of $S^{T} S$, and $\Lambda$ is the corresponding diagonal matrix of eigenvalues. To eliminate redundancy in the snapshots, the columns of $V$ corresponding to very small eigenvalues in $\Lambda$ are truncated. The matrix of eigenvalues $\Lambda$ is also resized to eliminate the rows and columns corresponding to the removed eigenvalues. If $Q-M$ columns of $V$ are truncated, the resulting reduced order mapping $\Phi$ will be an $N \times M$ matrix. The reduced-order mapping represents an isomorphism between $N$ and $M$ dimensional space. It determines the coordinates of $\mathbf{w}(t)$ in terms of the $M$ remaining basis functions, $\phi_{k}$.

The reduced-order mappings for each fluid variable are developed separately, and individual $S$ and $V$ arrays are collocated as blocks into a larger set of arrays, also denoted $S$ and $V$, to form

$$
\begin{aligned}
\boldsymbol{U}(t) & \approx \boldsymbol{U}_{0}+\Phi \widehat{\boldsymbol{U}}(t) \\
\Phi & =S V .
\end{aligned}
$$

These versions of Eqn.'s (16) and (17b), respectively, apply to the entire set of fluid variables.

POD of the discrete, panel position vector $w(x, t) \rightarrow$ $\boldsymbol{w}(t)$ and panel velocity vector $\boldsymbol{s}(t)=\dot{\boldsymbol{w}}(t)$ is accomplished in a similar manner as described above for the fluid system. Unlike the fluid POD basis functions, there is no base term subtracted from the snapshots when generating a structural POD basis.

\section{Volterra Methods}

Consider time-invariant, nonlinear, continuoustime, systems. Of interest is the response of the system about an initial state $\boldsymbol{X}(0)=\boldsymbol{X}_{0}$ due to an arbitrary input $u(t)$ (we take $u$ as a real, scalar input) for $t \geq 0$. As applied to these systems, Volterra theory ${ }^{1,26-28}$ yields the response

$$
\begin{aligned}
& \boldsymbol{X}(t)=\boldsymbol{h}_{0}+\int_{0}^{t} \boldsymbol{h}_{1}(t-\tau) u(\tau) d \tau \\
&+ \int_{0}^{t} \int_{0}^{t} \boldsymbol{h}_{2}\left(t-\tau_{1}, t-\tau_{2}\right) u\left(\tau_{1}\right) u\left(\tau_{2}\right) d \tau_{1} d \tau_{2} \\
&+\quad \sum_{n=3}^{N} \int_{0}^{t} . . \int_{0}^{t} \boldsymbol{h}_{n}\left(t-\tau_{1}, . ., t-\tau_{n}\right) \\
& u\left(\tau_{1}\right) . . u\left(\tau_{n}\right) d \tau_{1} . . d \tau_{n} .
\end{aligned}
$$

The Volterra series can be accurately truncated beyond the second-order term when a weakly nonlinear formulation is considered:

$$
\begin{gathered}
\boldsymbol{X}(t)=\boldsymbol{h}_{0}+\int_{0}^{t} \boldsymbol{h}_{1}(t-\tau) u(\tau) d \tau \\
+\int_{0}^{t} \int_{0}^{t} \boldsymbol{h}_{2}\left(t-\tau_{1}, t-\tau_{2}\right) u\left(\tau_{1}\right) u\left(\tau_{2}\right) d \tau_{1} d \tau_{2} .
\end{gathered}
$$

The assumption of a weakly nonlinear system is consistent with the emergence of limit-cycle oscillation of a 2-D aeroelastic system in transonic flow through a supercritical Hopf bifurcation. $^{29}$ For linear systems, only the first-order kernel is non-trivial, and there are no limitations on input amplitude.

The first- and second-order kernels are presented below in final form: ${ }^{5}$

$$
\begin{aligned}
\boldsymbol{h}_{1}\left(\tau_{1}\right) & =2 \boldsymbol{X}_{0}\left(\tau_{1}\right)-\frac{1}{2} \boldsymbol{X}_{2}\left(\tau_{1}\right) \\
\boldsymbol{h}_{2}\left(\tau_{1}, \tau_{2}\right) & =\frac{1}{2}\left(\boldsymbol{X}_{1}\left(\tau_{1}, \tau_{2}\right)-\boldsymbol{X}_{0}\left(\tau_{1}\right)-\boldsymbol{X}_{0}\left(\tau_{2}\right)(2)\right.
\end{aligned}
$$

In $(21), \boldsymbol{X}_{0}\left(\tau_{1}\right)$ is the time response of the system to a unit impulse applied at time 0 and $\boldsymbol{X}_{2}\left(\tau_{1}\right)$ is the time response of the system to an impulse of twice unit magnitude at time 0 . These response functions represent the memory of the system. If the system is linear, then $\boldsymbol{X}_{2}=2 \boldsymbol{X}_{0}$ and $\boldsymbol{h}_{1}=\boldsymbol{X}_{0}$, which is why the first-order kernel is referred to as the linear unit impulse response. The identification of the secondorder kernel is more demanding, since it is dependent on two parameters. Assuming $\tau_{2}>\tau_{1}$ in $(22), \boldsymbol{X}_{0}\left(\tau_{2}\right)$ is the response of the system to an impulse at time $\tau_{2}$.

Time is discretized with a set of time steps of equivalent size. Time levels are indexed from 0 (time 0 ) to $n$ (time $t$ ), and the evaluation of $\boldsymbol{X}$ at time level $n$ is denoted by $\boldsymbol{X}[n]$. The convolution in discrete time is

$$
\begin{aligned}
\boldsymbol{X}[n] & =\boldsymbol{h}_{0}+\sum_{k=0}^{N} \boldsymbol{h}_{1}[n-k] u[k] \\
& +\sum_{k_{1}=0}^{N} \sum_{k_{2}=0}^{N} \boldsymbol{h}_{2}\left[n-k_{1}, n-k_{2}\right] u\left[k_{1}\right] u\left[k_{2}\right](24)
\end{aligned}
$$

The linearized and nonlinear Volterra kernels can be transformed into linearized and nonlinear (bilinear) state-space systems that can be easily implemented into other disciplines such as controls and optimization. $^{5,27}$ For linear dynamics, state-space realization using the Eigensystem Realization Algorithm (ERA) has been used to generate linear, aeroelastic systems. ${ }^{16}$ Nonlinear system realization is an active area of research.

\section{System Realization}

The ERA method ${ }^{15}$ identifies a discrete, linear, time-invariant state-space realization of the form,

$$
\begin{aligned}
\boldsymbol{X}[n+1] & =A \boldsymbol{X}[n]+B \boldsymbol{u}[n] \\
\boldsymbol{Y}[n] & =C \boldsymbol{X}[n]
\end{aligned}
$$


using data from a complete ensemble of impulse responses. Initial state responses can be used in lieu of impulse responses, but we only consider impulse response data in this overview for simplicity. The systems realization procedure takes measurement data $\boldsymbol{Y}[n]$ from the free response of the system and produces a minimal state-space model $A, B$, and $C$ such that functions $\boldsymbol{Y}$ are accurately reproduced.

The free pulse response of linear, time-invariant, discrete systems is given by a function known as the Markov parameter,

$$
\boldsymbol{Y}_{\boldsymbol{m}}[n]=C A^{n-1} B .
$$

The superposition principle states that a system response to any arbitrary input can be obtained from a linear combination of impulse responses from that system. The generalized Hankel matrix of impulse responses is related to the Markov parameter by the superposition principle. The Hankel matrix is formed by windowing the impulse response data. A total of $K$ data points are provided at discrete time steps $n=1, \ldots K$, and the $r \times s$ matrix $H_{r s}$ is formed as follows,

$$
H_{r s}^{n-1}=\left[\begin{array}{ccc}
\boldsymbol{Y}_{\boldsymbol{m}}[n] & \ldots & \boldsymbol{Y}_{\boldsymbol{m}}[n+s-1] \\
\boldsymbol{Y}_{\boldsymbol{m}}[1+n] & \ldots & \boldsymbol{Y}_{\boldsymbol{m}}[1+n+s-1] \\
\vdots & \vdots & \vdots \\
\boldsymbol{Y}_{\boldsymbol{m}}[r-1+n] & \ldots & \boldsymbol{Y}_{\boldsymbol{m}}[r-1+n+s-1]
\end{array}\right]
$$

where $s$ is the total size of the data window, and $r$ is the number of time steps used to shift the data window. The choice of $r$ and $s$ is arbitrary as long as $r+s+n \leq K+2$.

The ERA method eliminates redundant data by using Singular Value Decomposition (SVD) on $\mathrm{H}_{r s}^{0}$,

$$
H_{r s}^{0}=P D Q^{T} \text {. }
$$

Unwanted state dimensionality is eliminated by truncating the elements of $P, D$, and $Q$ associated with very small singular values of $H_{r s}^{0}$. The number of states is reduced to a minimal number $q$. The number of observations $p$ and the number of forcing terms $m$ are known from the problem formulation. The dimension of the Markov parameter $\boldsymbol{Y}_{\boldsymbol{m}}[n]$ is $p \times m$. Algebra is used to recast Eqn. (26) in terms of the time shifted Hankel matrix $H_{r s}^{1}$, and the elements $P, D$, and $Q$. The state-space realization flows from this manipulation, and is as follows:

$$
\begin{aligned}
A & =D^{-\frac{1}{2}} P^{T} H_{r s}^{1} Q D^{-\frac{1}{2}} \\
B & =D^{\frac{1}{2}} Q^{T} E_{m} \\
C & =E_{p}^{T} P D^{\frac{1}{2}} .
\end{aligned}
$$

$E_{p}^{T}$ and $E_{m}^{T}$ are defined below:

$$
\begin{gathered}
E_{p}^{T}=\left[I_{p}, 0_{p}, \ldots, 0_{p}\right], \\
E_{m}^{T}=\left[I_{m}, 0_{m}, \ldots, 0_{m}\right],
\end{gathered}
$$

where $0_{p}$ and $0_{m}$ as the null matrices of order $p$ and $m$ respectively, and $I_{p}$ and $I_{m}$ are the identity matrices of order $p$ and $m$.

Since the discrete time step $\Delta t=t_{k+1}-t_{k}$ is constant, the continuous form of the discrete state-space realization (Eqn. (25)) is easily obtained. The continuous from, shown below, may require additional state dimensionality when the discrete realization has real, negative poles:

$$
\begin{aligned}
\dot{\boldsymbol{X}}(t) & =A \boldsymbol{X}(t)+B \boldsymbol{u}(t) \\
\boldsymbol{Y}(t) & =C \boldsymbol{X}(t) .
\end{aligned}
$$

\section{Aeroelastic ROM Development}

The full-order vector of fluid variables $\boldsymbol{U}(t)$ represents the spatially discretized flow field obtained from the full-system flow solver. POD provides a transformation $\Phi$ that maps $\boldsymbol{U}(t)$ to a low order vector of modal coefficients $\widehat{\boldsymbol{U}}(t)$ (from Eqn. 18a). The reducedorder fluid variable $\widehat{\boldsymbol{U}}(t)$ will be denoted $\boldsymbol{Y}_{f}$, which is the vector of outputs $\boldsymbol{Y}(t)$ (Eqn. (31)) .

A state-space model for $\boldsymbol{Y}_{f}$ can be obtained from impulse responses using the ERA method. Impulses for the fluid system use the plate position and velocity coefficients $\left(\boldsymbol{Y}_{s}\right)$ as the forcing terms. Each structural term is impulsed, and the fluid system response is generated using the full-order model. The time history of the impulse response is projected onto each of the POD basis functions to obtain the impulse response of the reduced-order fluid vector $\boldsymbol{Y}_{f}$. POD basis functions are obtained using the method of snapshots as described previously. The process is repeated for each structural mode, and the collection of impulse responses is used to generate a linear state-space model for the reduced order fluid system,

$$
\begin{aligned}
\dot{\boldsymbol{X}}_{f} & =A_{f} \boldsymbol{X}_{f}+B_{f} \boldsymbol{Y}_{s}, \\
\boldsymbol{Y}_{f} & =C_{f} \boldsymbol{X}_{f},
\end{aligned}
$$

where $\boldsymbol{X}_{f}$ is the state vector from the ROM realization, and $\boldsymbol{Y}_{s}$ represents the modal coefficients for the structural deformation.

The structural model from Eqn. (7b) is coupled to the reduced-order fluid model (Eqns. (32a) and (32b)) through the projected pressure term $\boldsymbol{P}$. The reducedorder variables, $\boldsymbol{Y}_{s}$, are obtained from the dynamic states $\boldsymbol{X}_{f}$ by the linear mapping $C_{f}$. Fluid pressure on the panel is extracted from $\boldsymbol{Y}_{s}$ using the portion of the reduced order mapping (Eqn. (18a)) that pertains to the moving boundary. Equation (5) is used to project the pressure values into $\boldsymbol{P}$. The mapping of reduced order fluid states to projected pressure is denoted $f_{P}$,

$$
\boldsymbol{P}=f_{P}\left(\boldsymbol{X}_{f}\right)
$$

Equation (33) is used to couple the structure and fluid dynamic state variables,

$$
\dot{\boldsymbol{Y}}_{s}=R_{s}\left(\boldsymbol{Y}_{s}, f_{P}\left(\boldsymbol{X}_{f}\right) ; \mu, \lambda, h / L\right) .
$$


Equations (32a) and (34) comprise the low order, aeroelastic ROM with linear fluid dynamics, and nonlinear plate dynamics.

The fluid and structural terms are grouped into arrays $\boldsymbol{Y}$ and $R$ as follows:

$$
\begin{aligned}
\boldsymbol{Y} & =\left[\begin{array}{c}
\boldsymbol{X}_{f} \\
\boldsymbol{Y}_{s}
\end{array}\right] \\
\widehat{R} & =\left[\begin{array}{c}
A_{f} \boldsymbol{X}_{f}+B_{f} \boldsymbol{Y}_{s} \\
R_{s}\left(\boldsymbol{Y}_{s}, f_{P}\left(\boldsymbol{X}_{f}\right) ; \mu, \lambda, h / L\right)
\end{array}\right] .
\end{aligned}
$$

The reduced-order, aeroelastic system is denoted as simply,

$$
\dot{\boldsymbol{Y}}=\widehat{R}(\boldsymbol{Y})
$$

Time Integration of the Aeroelastic System

The aeroelastic ROM (Eqn. (37)) is integrated in time with the two-time level, second-order accurate, Crank-Nicolson method:

$$
\begin{gathered}
\frac{\boldsymbol{Y}^{n+1}-\boldsymbol{Y}^{n}}{\Delta t}=\frac{1}{2}\left(\hat{R}^{n+1}+\hat{R}^{n}\right), \\
\mathcal{R} \equiv \boldsymbol{Y}^{n+1}-\frac{\Delta t}{2} \hat{R}^{n+1}-\boldsymbol{Y}^{n}-\frac{\Delta t}{2} \hat{R}^{n}=0 .
\end{gathered}
$$

At each time level, $\mathcal{Y} \equiv \boldsymbol{Y}^{n+1}$ is computed from Eqn. (39) using a chord technique with a time-frozen Jacobian

$$
\left(I-\frac{\Delta t}{2} \hat{J}_{o}\right)\left(\mathcal{Y}^{k+1}-\mathcal{Y}^{k}\right)=-\mathcal{R}\left(\mathcal{Y}^{k}\right),
$$

where $k$ is a subiteration index and $\hat{J}_{o}$ is the Jacobian of the reduced order aeroelastic system, evaluated for the base flow condition and $\boldsymbol{Y}_{s}=0$. A suitable number of subiterations are computed at each time step to obtain a good approximation to $\boldsymbol{Y}^{n+1}$; typically, 1-2 subiterations are generally sufficient to drive $\mathcal{R}$ to near machine zero. Since peak panel deflection is no more than $2 \%$ of panel length for the cases considered, the chord method is rapidly convergent. Prior to subiteration, $\mathcal{Y}$ is predicted from the explicit formula

$$
\mathcal{Y}=\boldsymbol{Y}^{n}+\Delta t \hat{R}^{n}
$$

\section{Results}

The results that follow consider supersonic free stream flow conditions at Mach 1.2, with sea level conditions. The Galerkin panel model contains 4 modes, for a total of 8 DOFs.

\section{Impulse Response of a Supersonic Fluid}

Impulsing the forcing term (or terms) of a truly linear system produces a response that is the building block necessary to recreate the system output from any arbitrary forcing function. Linear superposition allows the response of the system to be constructed in this fashion since any forcing function can be assembled from a series of impulses.

The supersonic flow field was essentially linear ${ }^{10}$ once LCO was fully developed. Shock waves formed at the ends of the panel, and although they varied in strength dynamically with the LCO, they were stationary, and the flow field between the shocks (directly over the panel) was linear for this case. However, impulsing the uniform, supersonic flow field with structural modes (and modal velocities) produced very nonlinear transient behavior. Figure 1 shows density contours of the flow 1.10802 nondimensional time units from the impulse of the fundamental structural velocity mode. The sudden appearance of a velocity profile on the
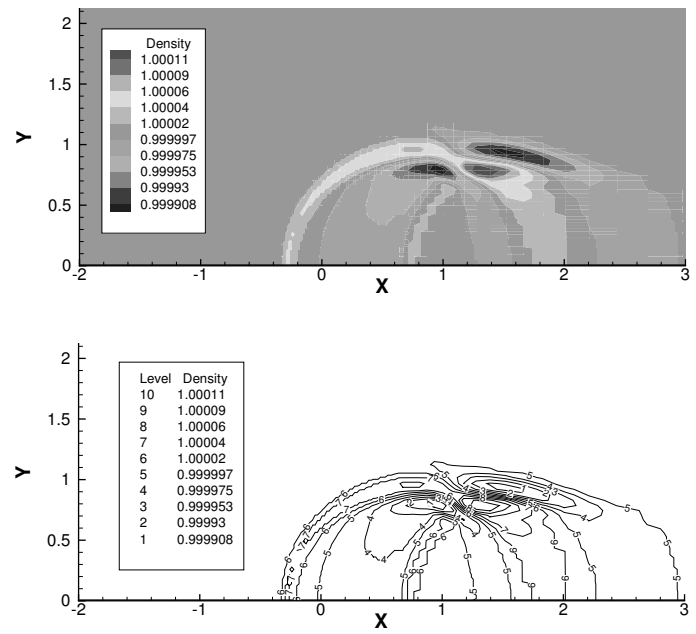

Fig. 1 Density contours 1.10802 time units after impulse

boundary (and its sudden removal one time step later) produced a shock wave of varying strength running the length of the panel. Early during the transient period, this shock welled upward away from the panel, and convected downstream. The convection of the shock, combined with the patterns of varied intensity produce the odd (but physical) spatial oscillation above the panel shown in Fig. 1. After about 2.5 time units, this pattern had both convected well beyond the end of the panel, and diffused into a more benign flow pattern. After 25 time units uniform flow was restored. The flow dynamics were essentially linear after the initial 2.5 time unit transient.

The results that follow will detail the usefulness of such impulse responses for generating a reduced-order fluid model. The linear portion of the impulse response time integrations contained some of the LCO flow field characteristics, otherwise none of the ROM options would have reproduced LCO. However, the impulse responses themselves would not produce POD basis functions capable of correctly modelling the LCO flow field. This suggests that the supersonic flow field was not truly linear. 


\section{Identification of Fluid Modes}

Fluid modes were obtained using POD as outlined previously. Aeroelastic fluid modes were obtained from a set of 100 snapshots. Snapshots of the fullorder, aeroelastic system were taken at equally spaced intervals, from start-up through 25 time units, using time integration of the full system, with $\lambda=25$. For all time integration cases that follow, the aeroelastic system was initialized with the base flow condition, and a small perturbation (height of 0.0001) in the fundamental panel position mode (denoted $a_{1}$ in Eqn. (7a). Snapshots were taken of primitive fluid variables $\rho, u$, $v$, and $E_{T}$, not the conserved variables given in Eqn. (8b). Primitive variables enable Galerkin projection for the fluid as a possible means for obtaining nonlinear terms ${ }^{13}$ in future analysis. At Mach 1.2, LCO required about 300 time units to become fully developed, and the small, 25 time unit training window was shown to be adequate in previous work. ${ }^{10,12}$

The results that follow refer to two cases. For the first case, the base flow term $\boldsymbol{U}_{0}$ from Eqn. (18a) consisted of uniform, free-stream conditions everywhere throughout the domain (referred to as slug flow). The second case considered steady state flow over the initial panel perturbation as the base flow term $\boldsymbol{U}_{0}$. For both cases, the first two modes for each fluid variable contained over $98 \%$ of the energy content, and system realization was performed using a total of $M=8$ fluid modes (2 modes per fluid variable). We also attempted to use the impulse response data as snapshots, in lieu of aeroelastic time integration. The same systems realization procedure was repeated to produce a Volterra-POD ROM for this third case, but this ROM did not correctly produce LCO. We documented our observations, and recommendations regarding this third approach in a separate section.

\section{System Realization}

We considered 8 POD modes, the dimensionality of $\boldsymbol{Y}_{f}$, which produced eight impulse responses for each forcing function. With 8 forcing terms in $\boldsymbol{Y}_{s}$, the total number of impulse responses numbered 64. Realization via the ERA process for each of the aeroelastic cases is detailed below.

\section{Uniform Base Flow}

A state-space realization of the form in Eqn. (25) was obtained using ERA for the slug-flow base case. The impulse amplitude was arbitrarily chosen to be 0.1. The full-system response to this impulse was sampled over 30 non-dimensional time units at a rate of $d t=0.015432$ for a total of $K=1944$ discrete data points. The fluid system impulse response was generated using the full-order model. The time history of the full-order impulse response was projected onto each of the POD basis functions to obtain the impulse response of the reduced-order fluid variable $\boldsymbol{Y}_{f}$. The data was windowed using $s=192$ and $r=100$. Every fifth data point was used in the realization algorithm providing data at the rate of $d t=0.07716$. These values of $d t, s$ and $r$ were chosen by trial and error to produce realizations whose impulse responses closely matched the data. The value of $m$ was 8 to match the number of forcing terms, and $p=8$ was chosen to match the number of ROM coefficients. The collection of impulse responses formed an $8 \times 8$ Markov parameter $\boldsymbol{Y}_{\boldsymbol{m}}[n]$ function from Eqn. (26). The number of states, $q=8$, was chosen to match the number of ROM coefficients, so SVD on the Hankel matrix formed from $\boldsymbol{Y}[n=1]$ was used to truncate all but the largest 8 singular values, yielding the matrices $P, D$, and $Q$. Equations (29a, 29b, 29c) were then used to generate a linear state-space model for the reduced-order fluid system,

$$
\begin{aligned}
\boldsymbol{x}[n+1] & =A \boldsymbol{x}[n]+B \beta \boldsymbol{u}[n], \\
\boldsymbol{y}[n] & =C \boldsymbol{x}[n],
\end{aligned}
$$

where $\beta$ was a scaling parameter that was used to calibrate the forcing amplitude.

The value of the scaling parameter $\beta=650$ was set by tuning the ROM results to the snapshot data. Theoretically, $\beta$ should have been the inverse of the impulse size $(\beta=1 / 0.1=10)$. The need for an order magnitude increase in $\beta$ reveals an inefficiency in projecting the impulsed flow-field onto the aeroelastic modes. Evidently, the impulsed flow-field contained structures not adequately represented in the aeroelastic modes, and a significant amount of flow energy was not captured in the projections used to compute the modal-impulse behavior. However, enough linear, aeroelastic information was resident in the impulsed flow-field for the Volterra-POD realization to produce correct results (with $\beta$ properly adjusted).
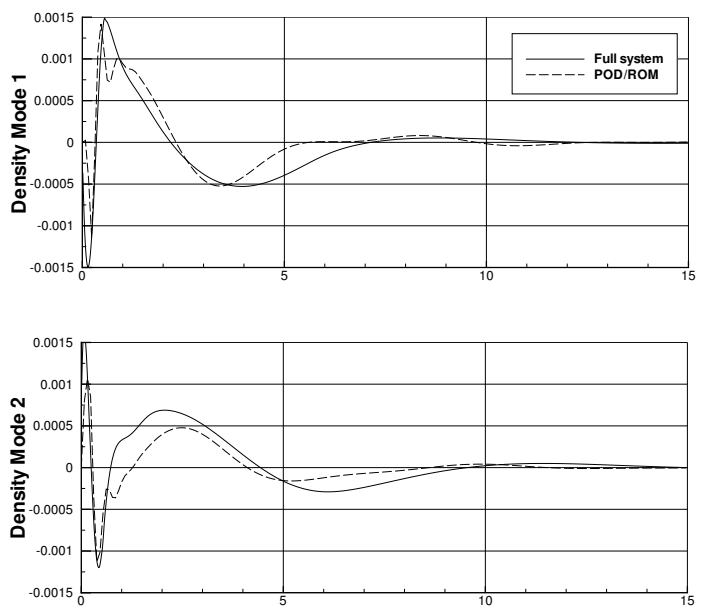

Fig. 2 Response of density modes to velocity term impulse, uniform base flow case

The impulse response of Eqns. (42a and 42b) was obtained with $\beta=1$. The impulse responses of the 
reduced-order system were in good agreement with the impulse responses from the full-order system used by ERA. The response of the two density modes to an impulse in the first panel velocity term $b_{1}$ within $\boldsymbol{Y}_{s}$ (defined in Eqn. (7a)) are shown in Fig. 2. The continuous form of Eqns. (42a and 42b) were obtained via a function call in MATLAB, which provided the matrices $A_{f}, B_{f}$ and $C_{f}$ for time integration of the aeroelastic model given in Eqn. (37).

\section{Steady-state Base Flow}

The same procedure was repeated, but the aeroelastic modes were computed using a steady-state base flow condition described earlier. For this realization, every $20^{t h}$ data point was used in the realization algorithm providing data at the rate of $d t=0.3086$. The same impulse amplitude of 0.1 was used for this case, and the full-system impulse response was sampled at the same rate. However, the impulses were added to the steady-state panel deflection for this case. The data was windowed using $s=47$ and $r=20$. Again, the value of $m$ was 8 to match the number of forcing terms, and $p=8$ was chosen to match the number of ROM coefficients. The scaling parameter from Eqn. (42a) was $\beta=800$, and was determined by tuning the ROM to the snapshot data.
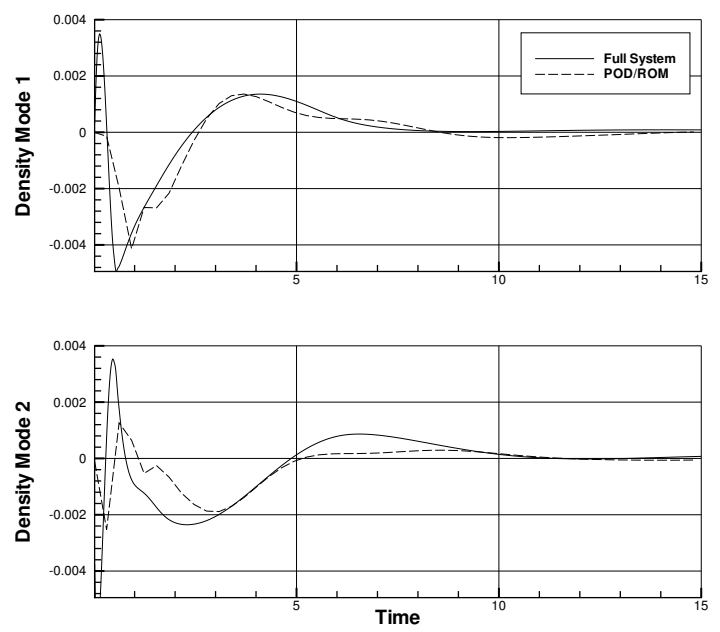

Fig. 3 Response of density modes to velocity term impulse, steady-state base flow case

The larger value of $d t$ eliminated much of the highfrequency transient, and focused ERA on the lowfrequency portion of the impulse response. This is reflected in the impulse response accuracy shown in Fig. 3, which considers the response of the two density modes to an impulse in the first panel velocity term $b_{1}$ within $\boldsymbol{Y}_{s}$ (defined in Eqn. (7a)). The first 3 seconds of the impulse response curve was not matched very closely by the ROM; however, these initial transients were not important to the LCO flow field.

\section{ROM Time History}

Both the slug-flow base case and the steady-state base case Volterra-POD ROMs, described above, were time-integrated using the aeroelastic training conditions of Mach 1.2 and $\lambda=25$. The results are shown in Fig. 4. Both cases correctly predicted LCO, but
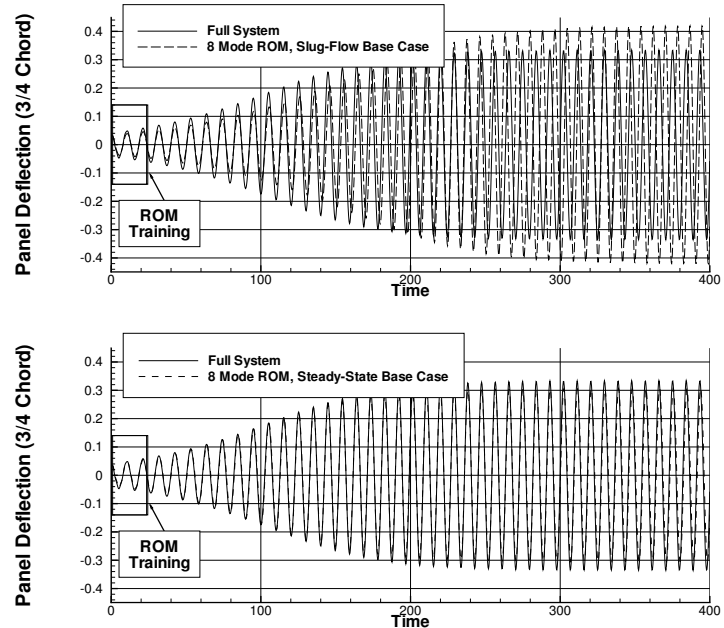

Fig. 4 Panel deflection $\left(w_{d} / h\right)$ time history, $\lambda=25$, Mach 1.2

the steady-state base case was more accurate in amplitude, frequency and phase than the slug-flow base case. The $3 / 4$ chord panel-amplitude was muted by $9 \%$ for the steady-state base case, and magnified by $25 \%$ for the slug-flow base case. The phase and frequency error were negligible for the steady-state base case, but the larger amplitudes of the slug-flow base case introduced a small increase in LCO frequency (resulting in an accumulating phase error).

We suspect the improvement in performance associated with the steady-state base flow was most likely due to the choice of data windowing parameters used in the ERA realization. We noticed no substantive differences in either the POD modes, or the impulse response of the full system flow-field between either case. Data windowing parameters were selected to provide a realization whose impulse response closely matched the original impulse data. As noted previously, reducing the size of $d t$ introduced high-frequency dynamics into the realization. The slug-flow base case was formed using a very small value of $d t$. Consequently the impulse response of the model (see Fig. 2) matched the initial transient in the data better than the steady-state base flow case (i.e. Fig. 3), which used a much larger $d t$. However, the high-frequency data in the impulse response was not germane to the large-time behavior of the aeroelastic system, and its inclusion resulted in a less accurate ROM under aeroelastic conditions. 


\section{ROM Robustness}

Both aeroelastic ROMs were time-integrated using a variety of panel dynamic pressure values $(\lambda)$. The intent was to explore the predictive accuracy of the Volterra-POD ROMs across a nonlinear parameter space. Both ROMs were trained at $\lambda=25$ (as described earlier), and robustness was defined as the ROM's ability to predict panel amplitude (at the $3 / 4$ chord position) in fully developed LCO across the parameter space, including non-LCO cases.

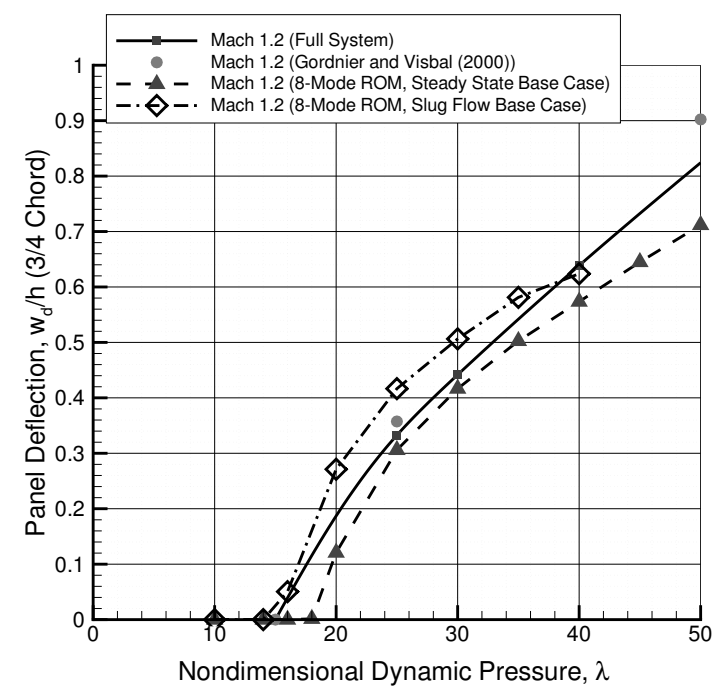

Fig. 5 Panel response verse dynamic pressure

The ROM results are compared with full system results, and results from the literature ${ }^{30}$ in Fig. 5. The steady-state base case ROM was better suited to large LCO amplitudes where the larger panel amplitudes excited a panel nonlinearity that corrupted the results of the slug-flow base case. Conversely, the use of slug flow as the base-flow term permited a more accurate prediction of LCO onset at the lower values of $\lambda$. A non-LCO solution for the slug-flow base case occurred when $\boldsymbol{Y}_{f}=[0]$ and $\boldsymbol{Y}_{s}=[0]$, but the use of steadystate flow over an initial, non-zero value of $\boldsymbol{Y}_{s}$ required a non-zero value of $\boldsymbol{Y}_{f}$ to produce $\boldsymbol{Y}_{s}=[0]$. The steady-state base case had difficulty producing this result. In addition, the small panel amplitudes near LCO-onset did not excite the high-frequency errors in the slug-flow base case that were evident at larger values of $\lambda$.

\section{Aeroelastic Structural Modes}

Aeroelastic structural modes were generated using 100 snapshots of the structural response obtained during the training of the fluid ROM. The structural snapshots corresponded exactly in time with the set of 100 snapshots used to construct the fluid ROMs. Snapshots were taken of the panel position and velocity vectors $(\boldsymbol{w}(t)$ and $\dot{\boldsymbol{w}}(t)$ respectively), and subspace projection $^{8}$ was used to form a reduced-order structural model. Subspace projection relied on the Galerkin panel model for time integration. The panel position and velocity from the Galerkin panel model were projected onto the POD basis functions at every step in the time integration. Subspace projection demonstrated the adequacy of the POD modes at capturing the dynamic panel behavior, while maintaining the nonlinearity of the panel dynamics.

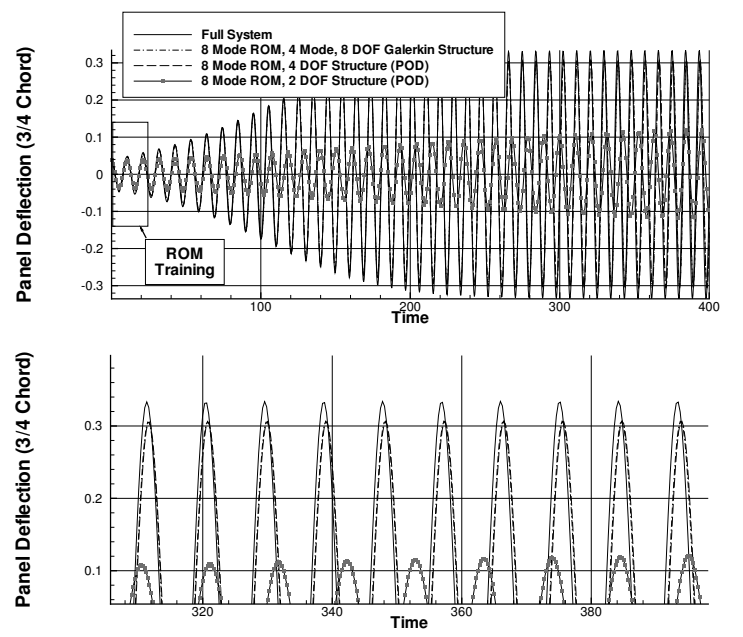

Fig. 6 Panel response $\left(w_{d} / h\right)$ with aeroelastic structural modes

The reduced-order structural model was tightly coupled with the steady-state base case fluid ROM and time-integrated using the parameter value $\lambda=25$. Figure 6 compares the results with the full system response, and the $\mathrm{POD} / \mathrm{ROM}$ results from Fig. 4 (for the steady-state base case). For clarity, the top, right-hand corner of the entire time response is expanded in the lower portion of Fig. 6. Two POD modes per structural variable (4 DOFs total) yielded essentially identical results to the 4 mode (8 DOF) Galerkin result. Further order-reduction greatly decreased the panel response. For this problem, the full-system structural model was very low order, and the additional order reduction from POD was immaterial. However, future application of this technique will involve very high-order, nonlinear structural models requiring order-reduction along with the fluid model. These results demonstrate that a single training event can produce adequate POD modes for both the fluid and structure.

\section{ROMs Using Impulse Response Modes}

As an excursion, the aeroelastic fluid modes were replaced with POD modes derived from the impulse responses of the full system. Forty snapshots were collected from each of the eight impulse responses generated by impulsing the elements of $\boldsymbol{Y}_{s}$. The snapshots were taken at even intervals over a time integration lasting 30 time units. Since there were 8 impulse responses, a total of 320 snapshots were generated. Over $98 \%$ of the flow energy was contained in the first 4 
modes for each of the 4 fluid variable. This suggests a 16 mode ROM would be adequate for the fluid. To test the adequacy of the POD basis, the full-system flow solution from time integration using $\lambda=25$ and Mach 1.2 was projected onto the POD basis during time integration. The modal amplitudes were examined and modes 6,7 and 8 in energy content had much greater contribution to the aeroelastic flow field than did the first 4 modes (note that the modes were normalized). This observation led us to consider 10 modes per fluid variable for ROM realization. ERA was used to synthesize a 40 mode ROM, which required 43 states after conversion to the continuous form. The Volterra-POD ROM was coupled with the Galerkin panel model as described previously.

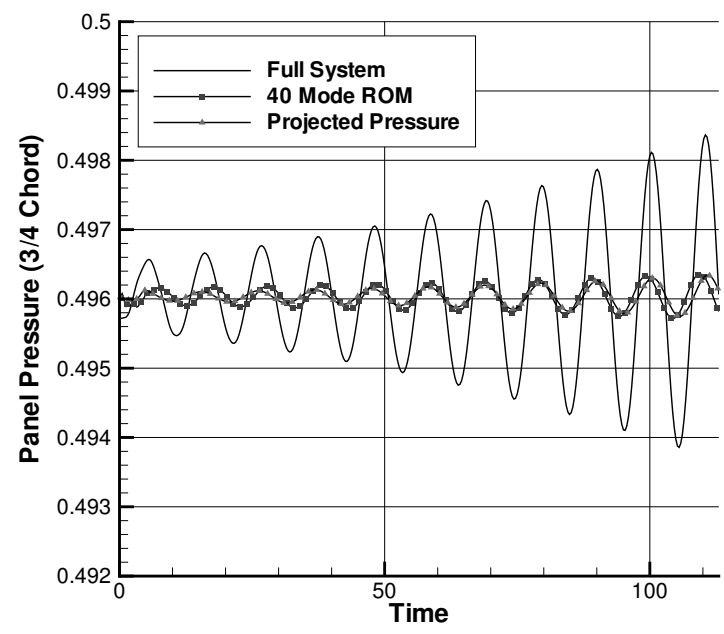

Fig. 7 Panel pressure degradation using impulse response modes

Unfortunately, the aeroelastic ROM produced a slowly divergent flutter instead of LCO. However, the panel pressures from the Volterra-POD ROM corresponded very well with the pressures obtained by projecting the full-system response onto the POD basis, and extracting the panel pressures (see Fig. 7). This further suggests that the POD basis derived from the impulse response data was not adequate for modeling the aeroelastic flow field. The accuracy of the ROM at representing the full system response was encouraging, and the frequency of unstable oscillations did match the LCO frequency. There is clearly some flowfield structure in the impulse response modes relevant to $\mathrm{LCO}$, but more investigation is required before these modes can be used to generate an accurate ROM for the supersonic case.

\section{Computational Performance}

The motivation for employing the POD-Volterra approach was to realize a computational performance improvement consistent with the reduction in the number of DOFs. Computational performance, summa- rized in Table 1, was assessed by measuring the wall clock time to provide fully developed $\mathrm{LCO}$ of the flow (400 time units with $\lambda=25$ ). All computations were run on a $800 \mathrm{MHz}$ Pentium-based, personal computer. The time-step used for the full system was based on a Courant-Friedrichs-Lewy (CFL) condition of 0.5 , which was the highest value allowing for stability of the second-order method. The reduced-order time integration used a time step size of 0.05 time units. The POD-Volterra ROM reduced compute time by fourorders-of-magnitude, and realized an improvement in performance consistent with the DOF reduction.

\begin{tabular}{ccc}
\hline \hline Flow Solver & Fluid DOFs & Wall Clock Time \\
\hline Full-order & 64400 & $22304 \mathrm{sec}$ \\
\hline POD-Volterra & 8 & $8.132 \mathrm{sec}$ \\
\hline \hline
\end{tabular}

Table 1 Computational performance

The cost of computing the state-space realization using ERA was small relative to the cost of a fullsystem analysis. Since there were eight forcing terms, eight additional runs of the full order solver were required to provide the impulse response data. Each 25 time unit impulse response runs required 1394 seconds wall-clock time, resulting in 11152 seconds of computer time for generating the impulse response data. An additional 25 time unit run was required for snapshot collection to generate the aeroelastic modes, bringing the total computer processing time to 12546 seconds, or roughly half of the computational cost associated with a single full-system time integration.

\section{Conclusions}

The Volterra-POD approach produced a stable and accurate aeroelastic ROM with four orders of magnitude reduction in problem order and computational expense. Aeroelastic modes were formed from snapshots obtained during the initial build-up of LCO, and with a fixed value of dynamic panel pressure. The full system model was impulsed using the structural velocity and position modes. The flow field was projected onto the aeroelastic modes to determine the modal amplitudes, and a linear, state-space realization for the fluid dynamics was synthesized from the modal impulse responses. The fluid and structural models were tightly coupled to form the aeroelastic ROM. Two cases were considered, one used uniform flow as a base term about which perturbations were computed by the reduced-order fluid model. The second case used steady-state flow over the initial panel deflection as the base-flow term. Both cases resulted in ROMs that correctly predicted LCO behavior over a wide parameter space; however, we concluded that uniform base flow was more desirable for predicting LCO onset. In addition, it was desirable to filter highfrequency information out of the impulse response data by computing the realization with relatively large time steps. 
Fluid modes obtained from snapshots of the impulsed flow-field were found to be inadequate for accurate reduced-order modeling of the supersonic, aeroelastic flow field. However, we only made an initial attempt to examine this approach, and additional research should be conducted before dismissing the technique completely.

Reduced-order modeling of the structure was also explored, and the results demonstrated that a single training event could produce adequate POD modes for both the fluid and structure. Future applications of this technique will involve very high-order, nonlinear structural models requiring order-reduction along with the fluid model.

\section{Acknowledgment}

The authors gratefully acknowledge the support of the Air Force Office of Scientific Research under grant 99VA01COR (Dr. Dean Mook, Program Manager).

\section{References}

${ }^{1}$ Volterra, V., Theory of Functionals and of Integral and Integro-Differential Equations, Dover Publications, Inc., New York, 1959.

${ }^{2}$ Loeve, M., Functions de Second Ordre, C. R. Academie des Sciences, Paris, France, 1945.

${ }^{3}$ Karhunen, K., Zur Spektral Theorie Stochastischer Prozesse, Ann. Acad. Sci. Fennicae, Ser, 1946.

${ }^{4}$ Holmes, P., Lumley, J., and Berkooz, G., Turbulence, Coherent Structures, Dynamical Systems and Symmetry, Cambridge University Press, 1996

${ }^{5}$ Silva, W. A., Discrete-Time Linear and Nonlinear Aerodynamic Impulse Responses for Efficient CFD Analyses, Ph.D. thesis, College of William \& Mary, December 1997.

${ }^{6}$ Silva, W. A., "Reduced-Order Models Based on Linear and Nonlinear Aerodynamic Impulse Responses," CEAS/AIAA/ICASE/NASA Langley International Forum on Aeroelasticity and Structural Dynamics, Williamsburg, VA, June 1999, pp. 369-379.

${ }^{7}$ Raveh, D., Levy, Y., and Karpel, M., "Aircraft Aeroelastic Analysis and Design Using CFD-Based Unsteady Loads," AIAA Paper 2000-1325, April 2000.

${ }^{8}$ Beran, P. and Silva, W., "Reduced Order Modeling: New Approaches for Computational Physics," AIAA 2001-0853, 39th Aerospace Sciences Meeting and Exhibit, Reno, NV, January 2001.

${ }^{9}$ Pettit, C. L. and Beran, P. S., "Reduced-Order Modeling for Flutter Prediction," AIAA 2000-1446-CP, 41st AIAA/ASCE/AHS/ASC Structural, Structural Dynamics and Matrerials Conference, Atlanta, GA. Accepted for publication in December 2001, International Journal for Numerical Methods in Engineering, April 2000.

${ }^{10}$ Beran, P. and Pettit, C., "Prediction of Nonlinear Panel Response Using Proper Orthogonal Decomposition," AIAA 2001-1292, 42nd Structural, Structural Dynamics, and Material Conference, Denver, CO, April 2001.

${ }^{11}$ Lucia, D. J., Beran, P. S., and King, P. I., "Reduced Order Modeling of an Elastic Panel in Transonic Flow," AIAA 20021594, 43rd AIAA/ASME/ASCE/AHS Structures, Structural Dynamics, and Materials Conference, Denver, CO, April 22-25 2002.

${ }^{12}$ Beran, P. S., Lucia, D. J., and Pettit, C. L., "Reduced Order Modeling of Limit-Cycle Oscillation for Aeroelastic Systems," IMECE 2002-32954, 2002 ASME International Mechanical Engineering Congress and Exposition, New Orleans, LA, November 17-22 2002.
${ }^{13}$ Lucia, D. J. and Beran, P. S., "Projeciton Methods for Reduced Order Models of Compressible Flows," Journal of Computational Physics, Vol. TBD, No. TBD, Accepted for publication, Jan 2003, pp. TBD.

${ }^{14}$ Lucia, D. J. and Beran, P. S., "Reduced Order Model Development Using Proper Orthogonal Decomposition and Volterra Theory," Submitted to the AIAA Journal, Vol. TBD, No. TBD, 2002, pp. TBD.

${ }^{15}$ Juang, J. N. and Pappa, R. S., "An Eigensystem Realization Algorithm for Modal Parameter Identification and Model Reduction," Journal of Guidance, Vol. 8, No. 5, 1984, pp. 620627.

${ }^{16}$ Silva, W. A. and Bartels, R. E., "Development of Reduced Order Models for Aeroelastic Analysis and Flutter Prediction Using the CFL3Dv6.0 Code," AIAA 2002-1594, 43rd AIAA/ASME/ASCE/AHS Structures, Structural Dynamics, and Materials Conference, Denver, CO, April 22-25 2002.

${ }^{17}$ Dowell, E. H., "Nonlinear Oscillations of a Fluttering Plate," AIAA Journal, Vol. 4, No. 7, July 1966, pp. 1267-1275.

${ }^{18}$ Sirovich, L., "Turbulence and the Dynamics of Coherent Structures. Part 1: Coherent Structures," Quarterly of Applied Mathematics, Vol. 45, n3, October 1987, pp. 561-571.

${ }^{19}$ Selvam, R. P. and Morton, S. A., "Computation of Nonlinear Viscous Panel Flutter," AIAA 98-1844, 39th Structural, Structural Dynamics, and Material Conference, Long Beach, CA, 20-23 April 1998.

${ }^{20}$ Tannehill, J. C., Anderson, D. A., and Pletcher, R. H., Computational Fluid Mechanics and Heat Transfer, Hemisphere Publishing Company, Washington DC, 1997.

${ }^{21}$ Stakgold, I., Green's Functions and Boundary Value Problems, John Wiley and Sons, Inc., Washington DC, 2nd ed., 1998.

${ }^{22}$ Sankar, L. N., Ruo, S. Y., and Malone, J. B., "Application of Surface Transpiration in Computational Aerodynamics," AIAA 86-0511, AIAA 24th Aerospace Sciences Meeting, Reno, NV, 1986.

${ }^{23}$ Lindsey, W. F. and Daley, B. N., "Effects of Compressibility on the Flow Past a Two-Dimensional Bump," Technical Note 2484, National Advisory Committee for Aeronautics, Langley Aeronautical Laboratory, Langley Field, VA, 1952.

${ }^{24}$ Anderson, J. D., Hypersonic and High Temperature Gas Dynamics, McGraw-Hill, 1989.

${ }^{25}$ Naylor, A. W. and Sell, G. R., Linear Operator Theory in Engineering and Science, Springer-Verlag, Inc., New York NY, 1982.

${ }^{26}$ Schetzen, M., The Volterra and Wiener Theories of Nonlinear Systems, John Wiley \& Sons, 1980.

${ }^{27}$ Rugh, W. J., Nonlinear Systems Thoery, The VolterraWeiner Approach, John Hopkins University Press, Baltimore MD, 1981.

${ }^{28}$ Bendat, J. S., Nonlinear System Analysis $\&$ Identification from Random Data, Wiley-Interscience, 1990.

${ }^{29}$ Beran, P. S., Huttsell, L. J., Buxton, B. J., Noll, C., and Osswald, G., "Computational Aeroelastic Techniques for Viscous Flow," CEAS/AIAA/ICASE/NASA Langley International Forum on Aeroelasticity and Structural Dynamics, Williamsburg, VA, June 22-25 1999.

${ }^{30}$ Gordnier, R. E. and Visbal, M. R., "Development of a Three-Dimensional Viscous Aeroelastic Solver for Nonlinear Panel Flutter," AIAA 2000-2337, Fluids 2000 Conference and Exhibit, Denver, CO, June 19-22 2000. 